\title{
Cardiotossicità da agenti antitumorali
}

\author{
Daniela Cardinale ${ }^{1}$, Alessandro Colombo ${ }^{1}$, Carlo Maria Cipolla ${ }^{1}$ \\ 1 Unità di Cardiologia, Istituto Europeo di Oncologia, IRCCS. Milano
}

\begin{abstract}
Chemotherapy-induced cardiotoxicity remains an unresolved problem strongly impacting the quality of life and the overall survival of cancer patients. The most typical form of cardiotoxicity, a dilated cardiomyopathy, usually becomes manifest late in the course of the disease and it is classically considered to be refractory to therapy. Detection of subclinical cardiac injury is crucial since it may facilitate early therapeutic measures. To detect cardiac damage, the most frequently adopted diagnostic approach is the monitoring of left ventricular ejection fraction by echocardiography or radionuclideangiocardiography; however, these methods utilized in clinical practice have low sensitivity and poor predictive value. Hence, other strategies, including an early detection of cardiotoxicity by biomarkers, have been proposed. The role of troponin I in identifying patients at risk of cardiotoxicity, and of angiotensin-converting enzyme inhibitors in preventing left ventricular ejection fraction reduction and cardiac events, is clearly emerging as an effective approach for the prevention of this complications. When chemotherapy-induced cardiomyopathy develops, however, no definite guidelines are currently adopted, and, although it is likely that angiotensin-converting enzyme inhibitors and beta-blockers may be highly effective, there is still some unjustified concern about using them in cancer patients.
\end{abstract}

Keywords: cardiotoxicity, chemotherapy-induced cardiomyopathy, heart failure, troponin

Cardiotoxicity of antitumor drugs

Pratica Medica \& Aspetti Legali 2009; 3(4): 155-161

\section{CASO CLINICO}

Il Signor G.A. è un paziente di 36 anni, ex fumatore, senza nessun precedente cardiovascolare, affetto da linfoma non Hodgkin a grandi cellule B. Nell'arco di un periodo di 16 mesi viene sottoposto a multipli trattamenti chemioterapici comprendenti farmaci antitumorali diversi, in associazione e ad alto dosaggio; poiché tali farmaci sono potenzialmente cardiotossici, viene eseguito il monitoraggio della funzione cardiaca durante il periodo di trattamento, mediante la valutazione ecocardiografica seriata della frazione di eiezione ventricolare sinistra (FEVS) (Tabella I).

Lultima valutazione avviene prima dell'ultimo ciclo di chemioterapia (CT) e mostra una FEVS an- cora nei limiti della normalità. Successivamente il paziente, che risiede in una regione diversa da quella del centro oncologico in cui ha effettuato la $\mathrm{CT}$, prosegue i controlli oncologici vicino al proprio domicilio. È in buone condizioni generali, non ha sintomi di richiamo cardiologico. Dall'ultimo ciclo di CT risulta libero da malattia e non esegue più controlli cardiologici ed ecocardiografici. Dopo 8 mesi viene ricoverato per la comparsa di dispnea a riposo: viene diagnosticata una cardiomiopatia (CMP) dilatativa/ipocinetica con severa riduzione della FEVS (21\%). Inizia una terapia cardiologica con inibitori dell'enzima di conversione dell'angiotensina (ACEI), digitale e diuretici; dopo poche settimane gli ACEI vengono sospesi per riscontro di ipotensione. Dopo 3 mesi viene nuovamente ricoverato per scom- 


\begin{tabular}{|ccc|}
\hline Mese & Schema CT & FEVS (\%) \\
\hline 1 & ACOD & \\
\hline 1 & CHOP & \\
\hline 3 & CTX & 61 \\
\hline 4 & VP16 & \\
\hline 4 & Mitoxantrone + melfalan & 67 \\
\hline 6 & R-ACOD X 4 & \\
\hline 9 & Rituximab 1 & 59 \\
\hline 9 & Rituximab 2 & \\
\hline 12 & ACOD X 2 & 57 \\
\hline 13 & ESHAP & \\
\hline 14 & ESHAP & 54 \\
\hline 15 & BEAM & \\
\hline
\end{tabular}

Tabella I. Schemi chemioterapici somministrati e variazione della FEVS ad essi correlata

$\mathrm{ACOD}=$ adriamicina, ciclofosfamide, vincristina, prednisone; $\mathrm{BEAM}$ $=\mathrm{BCNU}$, etoposide, ara-C e melphalan; $\mathrm{CHOP}=$ ciclofosfamide, doxorubicina, vincristina, prednisone; $C T X=$ ciclofosfamide; $E S H A P=$ etoposide, ara-C, cisplatino, desametasone; VP16 = etoposide

penso cardiaco (SC) acuto (FEVS 25\%). Dalla dimissione assume regolarmente una terapia cardiologica con ACEI, beta-bloccanti (BB) e diuretici. Attualmente, libero da malattia oncologica, presenta un discreta tolleranza allo sforzo (classe NYHA 2-3); la valutazione ecocardiografica continua tuttavia a evidenziare una severa riduzione della FEVS (35\%) dopo 2 anni di trattamento cardiologico.

\section{CARDIOTOSSICITÀ DA FARMACI CHEMIOTERAPICI}

La cardiotossicità rappresenta uno dei fattori che maggiormente limitano l'uso dei farmaci chemioterapici con un forte impatto sulla qualità di vita e sulla sopravvivenza del paziente, indipendentemente dal problema oncologico di base [1-4]. La rilevanza clinica della cardiotossicità è in continua ascesa come conseguenza del numero sempre crescente di pazienti che vengono sottoposti a CT, dell'introduzione continua di nuovi agenti antitumorali sempre più aggressivi ed efficaci, del loro maggiore utilizzo ad alte dosi e in combinazione, nonché del prolungamento della sopravvivenza dei pazienti oncologici trattati con CT [1-5].

\section{IMPLICAZIONI CARDIOLOGICHE}

Lo sviluppo di una CMP dilatativo/ipocinetica rappresenta la più frequente manifestazione di cardiotossicità da CT. Questa forma di CMP ha una fase di asintomaticità che può durare anche molti mesi, ed è ancora oggi ritenuta poco responsiva alla convenzionale terapia anti-SC $[2,6]$. Dati recenti della letteratura indicano che i pazienti trattati con CT costituiscono un gruppo di soggetti a più alto rischio di eventi cardiovascolari rispetto alla popolazione generale $[3,4]$. Più della metà dei pazienti trattati con antracicline (AC), che costituiscono la classe di chemioterapici più frequentemente utilizzata e di cui è ben noto il potenziale effetto cardiotossico, valutati anche dopo 10-20 anni dalla diagnosi oncologica iniziale, presentano alterazioni cardiache di diversa entità e vanno incontro a SC conclamato nel 5\% dei casi, e a fenomeni aritmici nel $40 \%$ dei casi; la mortalità cardiaca in questa categoria di pazienti risulta essere 8 volte più alta che nella popolazione generale $[7,8]$. In uno studio realizzato nel nostro istituto, che ha considerato una popolazione di più di 700 pazienti sottoposti a CT ad alte dosi e monitorati per un periodo medio di 3,5 anni, gli eventi cardiaci avversi hanno riguardato il $16 \%$ della popolazione [9].

\section{DIAGNOSI PRECOCE DELLA CARDIOTOSSICITÀ}

Al fine di identificare il danno cardiaco da CT in una fase ancora preclinica, viene raccomandato uno stretto monitoraggio della FEVS; tuttavia le linee guida dell'American Heart Association non specificano quando eseguire questi controlli, per quanto tempo e con che mezzo diagnostico [10]. Le raccomandazioni oncologiche esistenti suggeriscono il monitoraggio durante e dopo il trattamento antitumorale; tuttavia si riferiscono per lo più a popolazioni pediatriche, danno indicazioni disomogenee e non sembrano essere basate su chiare evidenze scientifiche [11]. In pratica, ad oggi, non esiste un approccio definito sul ruolo del monitoraggio della funzione cardiaca come prevenzione della cardiotossicità, specie dopo la fine della CT $[5,11]$. Poiché è noto infatti che questa forma di CMP si può manifestare anche dopo molti mesi dalla fine della terapia oncologica, risulta cruciale proseguire i controlli cardiologici per lungo tempo, anche se non è ancora noto quale sia il limite temporale oltre al quale si possa ritenere il paziente oncologico trattato con CT, non più "a rischio" di eventi cardiovascolari.

Inoltre, la maggior parte dei metodi di monitoraggio utilizzati nella pratica clinica (ecocardiografia, angiografia con radionuclidi) hanno mostrato una bassa accuratezza diagnostica e uno scarso pote- 
re predittivo [11]. Il semplice monitoraggio della FEVS non sembra, infatti, sufficientemente sensibile e specifico nel prevedere lo sviluppo di disfunzione cardiaca dopo CT ed è in grado di identificare il danno cardiaco solo quando esso ha già avuto ripercussioni funzionali, impedendo in questo modo ogni possibile strategia di prevenzione. Di conseguenza, vi è una forte esigenza, condivisa da oncologi e cardiologi, di nuovi strumenti diagnostici, non invasivi, sensibili, poco costosi, e facilmente ripetibili, che consentano di stratificare precocemente il rischio di cardiotossicità nei pazienti trattati con CT.

\section{Utilizzo di marker biochimici: troponina I (TNI)}

Se il ruolo della troponine nella diagnosi e nella stratificazione prognostica dei pazienti con sindromi coronariche acute è ormai ampiamente consolidato, la loro applicazione in campo oncologico nella diagnosi precoce del danno cardiaco da CT e nella stratificazione del rischio cardiaco a breve e a lungo termine è di più recente introduzione [9,12-15]. Nei nostri primi studi, condotti su una popolazione di più di 400 pazienti affetti da neoplasie molto aggressive, trattati con CT ad alto dosaggio, è emerso che la TNI, misurata subito dopo la fine dell'infusione dei farmaci antitumorali, sia in grado di fornire importanti informazioni dal punto di vista sia diagnostico che prognostico [13,14]. Il monitoraggio durante il primo anno di follow-up ha mostrato come solo nei pazienti con valori patologici di TNI (35\% dei pazienti trattati) si possa osservare una riduzione della FEVS (già significativa a 3-4 mesi dopo la fine della $\mathrm{CT}$ ), con una stretta correlazione tra il valore di TNI e il massimo calo funzionale $(r=-0,87$; $p<$ 0,0001 ) [13]. In pratica, la valutazione della TNI in questi pazienti fornisce due tipi di informazione: una di tipo qualitativo, e cioè indica quali pazienti sono a maggior rischio di sviluppare nel tempo una disfunzione cardiaca; e una di tipo quantitativo: tanto maggiore è il rialzo della TNI tanto più importante sarà l'entità del calo funzionale tardivo. Il dato decisamente innovativo è che questo marker cardiaco è in grado di identificare i pazienti a rischio di cardiotossicità in una fase estremamente precoce (subito dopo la CT), quando non sono ancora rilevabili criteri clinici (sintomi) e/o strumentali (all'elettrocardiogramma o all'ecocardiogramma) di cardiotossicità. Il pattern di rilascio della TNI sembra inoltre essere in grado di predire non solo il rischio di disfunzione ventricolare sinistra, ma anche quello di eventi cardiaci maggiori. In uno studio condotto nel nostro istituto, che ha incluso più di 700 pazienti oncologici trattati con CT ad alte dosi, e monitorati per un lungo periodo dopo la fine della CT (follow-up medio 3,5 anni) è emerso che nei pazienti senza incremento dei valori di TNI non si è riscontrata una riduzione significativa della FEVS e vi è stata una bassa incidenza di eventi cardiaci (1\%). Al contrario, la maggior parte degli eventi cardiaci è stata osservata nei pazienti con positivizzazione precoce della TNI e, in particolar modo, in quei pazienti in cui l'aumento plasmatico di questo marker è persistito per almeno un mese $(87 \%$; $<<0,001)$ [9].

L'alto valore predittivo negativo della TNI (99\%) [9] consente di discriminare tra pazienti a più alto rischio di sviluppare cardiotossicità e pazienti con una buona prognosi cardiologica. In particolar modo la TNI permette di identificare con sicurezza i pazienti a basso rischio, che rappresentano la maggior parte dei soggetti sottoposti a CT $(70 \%)$, consentendo di riservare le risorse necessarie per una stretta sorveglianza cardiologica ai soli pazienti nei quali la TNI ha svelato l'esistenza e la persistenza di un danno cardiaco subclinico dopo CT. Dato che queste informazioni prognostiche vengono fornite in uno stadio precoce, molto prima cioè di poter identificare il danno funzionale con tecniche strumentali quali l'ecocardiografia e molto prima della comparsa dei sintomi, è possibile mettere in atto, nei soli pazienti ad alto rischio, strategie terapeutiche mirate in una fase in cui il danno cardiaco è ancora potenzialmente prevenibile.

\section{PREVENZIONE FARMACOLOGICA: RUOLO DEGLI ACE-INIBITORI}

Gli ACEI sono in grado di rallentare la progressione della disfunzione ventricolare sinistra in numerosi contesti clinici [16]. Dati relativi a modelli animali indicano che il sistema renina-angiotensina tissutale cardiaco giochi un ruolo importante nello sviluppo e nella progressione della CMP indotta dalle $\mathrm{AC}$ e che un trattamento con ACEI, subito dopo la $\mathrm{CT}$, è in grado di esercitare un effetto protettivo $[17,18]$. Sulla base di queste osservazioni, abbiamo valutato se un trattamento con ACEI fosse in grado di prevenire lo sviluppo di cardiotossicità in 114 pazienti oncologici ad alto rischio, selezionati in base all'evidenza di incremento di valori di TNI subito dopo la CT e randomizzati al trattamento o meno con enalapril [19]. Durante il successivo monitoraggio (12 mesi), abbiamo osservato una significativa riduzione della FEVS (riduzione $>10$ punti percentuali, associata a una riduzione al di sotto del limite della normalità $(50 \%)$ nel $43 \%$ dei pazienti non trattati e in nessuno dei pazienti trattati con enalapril $(p<0,001)$. L'incidenza di eventi cardiaci 
durante il follow-up è stata significativamente più elevata nei pazienti non trattati $(52 \%$ vs $2 \%$; p < $0,001)$. Nei soggetti trattati con enalapril, la percentuale di pazienti con evidenza di valori persistentemente elevati di TNI durante il follow-up è andata rapidamente riducendosi sino ad azzerarsi dopo 2 mesi di trattamento; nei pazienti non trattati, invece, il numero dei pazienti ancora "positivi" si è ridotto molto più lentamente nel tempo e valori patologici di TNI erano ancora riscontrabili in 3 pazienti al termine del follow-up [19]. Questo diverso pattern di rilascio della TNI nelle due popolazioni di studio suggerisce che il danno cardiaco indotto dalla CT sia in grado di innescare un fenomeno on-going di progressiva perdita di miociti che si mantiene nel tempo, il cui effetto cumulativo si traduce in un deterioramento tardivo della funzione contrattile globale. Qualsiasi sia il meccanismo coinvolto, questi dati sembrano indicare che enalapril è in grado di "spegnere" il rilascio di TNI, prevenendo lo sviluppo di disfunzione cardiaca tardiva. Tutto ciò sul piano clinico si traduce in una riduzione degli eventi cardiaci avversi a lungo termine [19].

\section{DIFFERENZE CON ALTRE STRATEGIE DI PREVENZIONE}

Molteplici strategie sono state proposte nel tentativo di prevenire o ridurre la cardiotossicità $[20,21]$. Tra queste, le variazioni nello schema di somministrazione della CT, la limitazione della dose totale di AC, l'utilizzo di analoghi meno cardiotossici delle AC e di cardioprotettori (carvedilolo, valsartan, agenti antiossidanti, dexrazoxane, eritropoietina, ecc.) [20,21]. Tuttavia, ognuno di questi approcci presenta delle importanti limitazioni, tra le quali la possibile compromissione del successo clinico della CT e i costi elevati. Infine, la limitazione maggiore, comune a tutte queste strategie, è che tutti i pazienti trattati con CT devono essere indiscriminatamente considerati, con un rapporto costo/beneficio estremamente elevato. Viceversa, l'aspetto innovativo del nostro approccio è che la TNI ci consente di selezionare i pazienti più a rischio di sviluppare cardiotossicità tardiva $(25-30 \%$ nelle nostre popolazioni di studio) per i quali pianificare una terapia preventiva, escludendo da un trattamento profilattico la maggior parte dei pa-

\begin{tabular}{|c|c|c|c|c|c|c|c|c|c|c|c|}
\hline Terapia & Autore & Anno & $\begin{array}{l}\text { Pz } \\
\text { (n) }\end{array}$ & $\begin{array}{c}\text { Età } \\
\text { media } \\
\text { (aa) }\end{array}$ & $\begin{array}{l}\text { Tipo di } \\
\text { studio }\end{array}$ & $\begin{array}{c}\text { t-t-t } \\
\text { medio } \\
\text { (mesi) }\end{array}$ & $\underset{\text { (mesi) }}{\text { FU }}$ & $\begin{array}{c}\text { B } \\
\text { FEVS } \\
(\%)\end{array}$ & $\begin{array}{c}\text { F } \\
\text { FEVS } \\
(\%)\end{array}$ & $\begin{array}{l}\text { Evento } \\
\text { riportato }\end{array}$ & $\begin{array}{c}\text { Dose } \\
\text { media AC } \\
(\mathrm{mg} / \mathrm{mq})\end{array}$ \\
\hline Dig + Diur & Lefrak [23] & 1973 & 2 & 43 & $\mathrm{CR}$ & 1 & 1 & ND & ND & $M C$ & 772 \\
\hline Dig + Diur & Cohen [24] & 1982 & 1 & 38 & CR & 12 & 8 & 23 & 64 & $\downarrow$ sintomi & 475 \\
\hline Dig + Diur & Haq [25] & 1985 & 43 & 55 & $\mathbf{R}$ & 38 & 48 & ND & ND & MC, SC & 450 \\
\hline Dig + Diur + ACEI & Saini [26] & 1987 & 3 & 49 & $\mathrm{CR}$ & 4 & $12-16$ & 20 & 48 & $\begin{array}{l}\downarrow \text { sintomi } \\
\uparrow \text { FEVS }\end{array}$ & 450 \\
\hline $\begin{array}{l}\text { Dig + Diur* } \\
\text { Dig + Diur + ACEI }{ }^{\dagger}\end{array}$ & Jensen [27] & 1996 & 9 & 58 & $P$ & $\begin{array}{c}4 \\
12\end{array}$ & 26 & 27 & 47 & $M C, S C$ & 500 \\
\hline $\begin{array}{l}\text { Dig + Diur + } \\
\text { ACEI* }^{*} \\
\mathrm{BB}^{\dagger}\end{array}$ & Fazio [28] & 1998 & 1 & 35 & $\mathrm{CR}$ & 1 & 12 & 14 & 45 & $\downarrow$ sintomi & 500 \\
\hline $\begin{array}{l}B B \\
B B+A C E I\end{array}$ & Noori [29] & 2000 & $\begin{array}{l}2 \\
6\end{array}$ & 51 & $\mathrm{R}$ & ND & 32 & 28 & 41 & $\uparrow$ FEVS & ND \\
\hline $\begin{array}{l}\text { Dig + Diur* } \\
\text { Dig + Diur + ACEI }\end{array}$ & Jensen [30] & 2002 & 10 & 54 & $P$ & $\begin{array}{c}4 \\
12\end{array}$ & 30 & 27 & 41 & SC & 500 \\
\hline $\begin{array}{l}\mathrm{BB} \\
\mathrm{BB}+\mathrm{ACEI}\end{array}$ & Mukai [31] & 2004 & $\begin{array}{l}3 \\
2\end{array}$ & 53 & CR & 34 & 27 & 37 & 53 & $\begin{array}{l}\uparrow \text { FEVS } \\
\uparrow \text { NYHA }\end{array}$ & ND \\
\hline $\begin{array}{l}\text { ACEI } \\
\mathrm{ACEI}+\mathrm{BB}\end{array}$ & Tallaj [32] & 2005 & $\begin{array}{l}10 \\
15\end{array}$ & 47 & $\mathbf{R}$ & 77 & 70 & 25 & 34 & $\mathrm{MC}, \mathrm{MI}, \mathrm{TC}$ & 469 \\
\hline $\mathrm{ACEI}+\mathrm{BB}$ & Tabet [33] & 2006 & 1 & 52 & CR & 4 & 8 & ND & 30 & SC & 926 \\
\hline
\end{tabular}

Tabella II. Trattamento della cardiomiopatia da antracicline (AC): studi clinici

$\mathrm{ACEI}=$ inibitori dell'enzima di conversione dell'angiotensina; $\mathrm{B}=$ basale; $\mathrm{BB}=$ beta-bloccanti; $\mathrm{CR}=$ case report; $\mathrm{Dig}=$ digitale;

Diur = diuretici; $F=$ finale; FEVS = frazione d'eiezione ventricolare sinistra; $F U=$ follow-up; $M C=$ morte per causa cardiaca;

$\mathrm{MI}=$ morte improvvisa; $\mathrm{ND}=$ non disponibile; $\mathrm{NYHA}=$ New York Heart Association; $\mathrm{P}=$ prospettico; $\mathrm{R}=$ retrospettivo;

$\mathrm{SC}=$ scompenso cardiaco; $\mathrm{t}-\mathrm{t}-\mathrm{t}=$ time-to-treatment; $\mathrm{TC}=$ trapianto cardiaco

*terapia di prima linea; ${ }^{\dagger}$ terapia di seconda linea 
zienti, con un'accurata personalizzazione delle indicazioni e un migliore utilizzo delle risorse complessive di cura.

\section{TRATTAMENTO}

La CMP da AC viene considerata refrattaria alla terapia medica convenzionale, e, di conseguenza, viene associata a una prognosi particolarmente negativa, con un tasso di mortalità a due anni del $60 \%$ [22]. I dati relativi alla storia naturale di questa malattia e alla sua risposta alla terapia sono, tuttavia, aneddotici o ricavati da vecchi studi, in cui la terapia per lo SC era basata sul solo utilizzo di digitale e diuretici [23-25]. In realtà, questi pazienti sono sempre stati sistematicamente esclusi dai grandi studi clinici prospettici che hanno analizzato l'efficacia di farmaci quali gli ACEI e i BB e la risposta della CMP da AC alla moderna terapia per lo SC non è mai stata valutata se non in studi che hanno incluso un esiguo numero di pazienti, per lo più retrospettivi e senza endpoint predefiniti. Mettendo insieme tutti i dati pubblicati, è possibile ottenere una casistica complessiva di 108 pazienti adulti, da un totale di 11 pubblicazioni (6 case report e 5 studi clinici) (Tabella II) [23-33].

In tutti gli studi la terapia è stata iniziata solo dopo la comparsa dei sintomi di SC. Quarantasei pazienti (43\%) sono stati trattati con digitale e diuretici, e 32 (30\%) con differenti ACEI (enalapril nella maggioranza dei casi); tra i pazienti trattati con ACEI, solo 13 hanno ricevuto questi farmaci come prima linea di trattamento. Infine, solo 5 pazienti (5\%) sono stati trattati con soli BB (carvedilolo nella maggior parte dei casi) e solo 25 pazienti (23\%) hanno ricevuto l'associazione di queste due classi di farmaci. Altrettanto limitati sono i dati sulla prognosi a lungo termine dei pazienti affetti da questa forma di CMP, sia trattati che non trattati. Ad oggi, non esistono ancora indicazioni basate su chiare evidenze cliniche per la gestione di questa forma di CMP, né esistono linee guida specifiche per questa problematica clinica.

Va sottolineato inoltre che, a causa della particolare eziologia e della diversa distribuzione d'età di questo tipo di CMP, quando confrontata con le più frequenti forme su base ischemica o idiopatica, esiste ancora oggi una certa perplessità riguardo l'impiego di ACEI e BB in questa categoria di pazienti. Infatti, esiste ancora oggi il dubbio, specie tra gli oncologi, se questo tipo di approccio, pur raccomandato dalle linee guida cardiologiche internazionali, possa essere direttamente trasferito a questo particolare ambito clinico, con simili benefici a lungo termine [8]. Una caratteristica ingannevole della disfunzione cardiaca da AC è il fat- to che possa rimanere asintomatica anche per lungo tempo [8]. Per tale ragione, molti Autori hanno suggerito solo dei programmi di monitoraggio mirati alla ricerca dei casi di SC sintomatico, e di fatto vengono trattati solo i pazienti con disfunzione sintomatica $[23,32]$. Un punto cruciale e ancora dibattuto è se trattare i pazienti asintomati$\mathrm{ci}$, in cui la disfunzione ventricolare sinistra viene evidenziata solo occasionalmente attraverso esami di screening routinari, ed eventualmente in che modo. Ad oggi non esiste un consenso generale su cosa debba essere fatto per arrestare la progressione della CMP da AC, e di conseguenza non esistono raccomandazioni basate su chiare evidenze cliniche che aiutino il cardiologo a gestire questo tipo di CMP, sia nella fase asintomatica che sintomatica.

I dati di un nostro recente studio, suggeriscono che il tempo intercorso tra la fine della CT e l'inizio del trattamento anti-SC con ACEI e, quando tollerato, con BB è una variabile cruciale per il recupero della disfunzione cardiaca [34]. Infatti, la probabilità di ottenere un completo recupero della FEVS è più alto nei pazienti in cui il trattamento cardiologico viene iniziato entro due mesi dalla fine della CT. Dopo questo limite di tempo, tuttavia, questa probabilità si riduce progressivamente sino a esaurirsi completamente dopo 6 mesi.

Sulla base di questi dati, possiamo ipotizzare che la scarsa risposta alla terapia anti-SC descritta nella maggior parte degli studi pubblicati in passato fosse verosimilmente legata a un scarso uso dei moderni farmaci anti-SC, come gli ACEI e i BB, e al lungo (> 12 mesi) tempo intercorso tra la fine della CT e l'inizio del trattamento cardiologico, che veniva iniziato verosimilmente quando il danno cardiaco non era più reversibile. Questo dato enfatizza l'importanza strategica di una diagnosi precoce del danno cardiotossico e indica che un approccio aggressivo basato sull'associazione di ACEI e BB dovrebbe essere sempre preso in considerazione e tentato in tutti i casi di CMP indotta dalla CT.

\section{CONCLUSIONI}

La cardiotossicità indotta dai farmaci antitumorali rappresenta un problema irrisolto, fortemente impattante sulla qualità di vita e sulla sopravvivenza dei pazienti oncologici. Le principali strategie per minimizzare la cardiotossicità rimangono la diagnosi precoce dei pazienti ad alto rischio e il trattamento profilattico tempestivo.

La prevenzione della CMP da CT mentre si sta ancora trattando il tumore costituisce l'obiettivo ottimale. Pertanto, la funzione cardiaca dovrebbe es- 
sere sempre monitorata nei pazienti che ricevono una trattamento chemioterapico potenzialmente cardiotossico, per poter individuare precocemente eventuali anomalie cardiache ancora potenzialmente reversibili. L'assenza di chiare linee guida basate sull'evidenza si traduce nella realtà nel fatto che questo monitoraggio viene disatteso nei pazienti asintomatici e nei pazienti con remissione completa dalla malattia oncologica. Gli oncologi e i cardiologi dovrebbero pianificare insieme il monitoraggio di questi pazienti, dal momento che certe decisioni terapeutiche, se non condivise, possono favorire lo shift del paziente da una malattia a un'altra a prognosi altrettanto sfavorevole.

Il ruolo dei marker cardiaci nell'identificare i pazienti a rischio di cardiotossicità e degli ACEI nel prevenire la riduzione della FEVS e degli eventi cardiaci sta chiaramente emergendo come un approccio efficace per la prevenzione di questa complicanza. Per il suo trattamento non vengono, al momento attuale, adottate linee guida definite, e sebbene è verosimile che ACEI e BB possano essere molto efficaci, c'è ancora una preoccupazione ingiustificata circa il loro uso nei pazienti oncologici.

\section{BIBLIOGRAFIA}

1. Menna P, Salvatorelli E, Minotti G. Cardiotoxicity of antitumor drugs. Chem Res Toxicol 2008; 21: 978-89

2. Yeh ET, Tong AT, Lenihan DJ, Yusuf SW, Swafford J, Champion C et al. Cardiovascular complications of cancer therapy. Diagnosis, pathogenesis and management. Circulation 2004, 109: 3122-31

3. Robinson LL, Green DM, Hudson M, Meadows AT, Mertens AC, Packer RJ et al. Long-term outcomes of adult survivors of childhood cancer. Cancer 2005; 104: 2557-64

4. Jones LW, Haykowsky MJ, Swartz JJ, Douglas PS, Mackey JR. Early breast cancer therapy and cardiovascular injury. J Am Coll Cardiol 2007; 50: 1435-41

5. Carver JR, Shapiro CL, Ng A, Jacobs L, Schwartz C, Virgo K et al; ASCO Cancer Survivorship Expert Panel. American Society of Clinical Oncology clinical evidence review on the ongoing care of adult cancer survivors: cardiac and pulmonary effects. J Clin Oncol 2007; 25: 3991-4008

6. Barry E, Alvarez JA, Scully RE, Miller TL, Lipshultz SEl. Anthracycline-induced cardiotoxicity: course, pathophysiology, prevention and management. Expert Opin Pharmacother 2007; 8: 1039-58

7. Van den Belt-Dusebout AW, Nuver J, de Wit R, Gietema JA, ten Bokkel Huinink WW, Rodrigus PT et al. Long-term risk of cardiovascular disease in 5-year survivors of testicular cancer.J Clin Oncol 2006; 24: 467-75

8. Silber JH, Cnaan A, Clark BJ, Paridon SM, Chin AJ, Rychik J et al. Enalapril to prevent cardiac function decline in long-term survivors of pediatric cancer exposed to anthracyclines. J Clin Oncol 2004; 22: 820-8

9. Cardinale D, Sandri MT, Colombo A, Colombo A, Boeri M, Lamantia G et al. Prognostic value of Troponin I in cardiac risk stratification of cancer patients undergoing high-dose chemotherapy. Circulation 2004; 109: 2749-54

10. Hunt SA, Albraham WT, Chin MH, Feldman AM, Francis GS, Ganiats TG et al. ACC/AHA 2005 guideline update for the diagnosis and management of chronic heart failure in the adult: a report of the American College of Cardiology/American Heart Association Task Force on Practice Guidelines. Circulation 2003; 108: 1146-62

11. Altena R, Perik PJ, van Veldhuisen DJ, de Vries EGE, Gietema JA. Cardiovascular toxicity caused by cancer treatment: strategies for early detection. Lancet Oncol 2009; 10: 391-9

12. Lipshultz SE, Rifai N, Sallan SE, Lipsitz SR, Dalton V, Sacks DB et al. Predictive value of cardiac troponin T in pediatric patients at risk for myocardial injury. Circulation 1997; 96: 2641-8

13. Cardinale D, Sandri MT, Martinoni A, Tricca A, Civelli M, Lamantia G et al. Left ventricular dysfunction predicted by early troponin I release after high-dose chemotherapy.J Am Coll Cardiol 2000; 36: 517-22

14. Cardinale D, Sandri MT, Martinoni A, Borghini E, Civelli M, Lamantia G et al. Myocardial injury revealed by plasma troponin I in breast cancer treated with high-dose chemotherapy. Ann Oncol 2002; 13: 710-5

15. Auner HW, Tinchon C, Linkesch W, Tiran A, Quehenberger F, Link H et al. Prolonged monitoring of troponin T for detection of anthracycline cardiotoxicity in adults with hematological malignancies. Ann Hematol 2003; 82: 218-22

16. Lopez-Sendon J, Swedberg K, McMurray J, Tamargo J, Maggioni AP, Dargie H et al. Expert consensus document on angiotensin converting enzyme inhibitors in cardiovascular disease. The task force on ACE-inhibitors of the European Society of Cardiology. Eur Heart J 2004; 25: 1454-70

17. Okumura K, Jin D, Takai S, Miyazaki M. Beneficial effects of angiotensin-converting enzyme inhibition in adryamicin-induced cardiomyopathy in hamsters. Jpn J Pharmacol 2002; 88: 183-8

18. Tokudome T, Mizushige K, Noma T, Manabe K, Murakami K, Tsuji T et al. Prevention of doxorubicin (adriamycin)-induced cardiomyopathy by simultaneous administration of angiotensin-converting enzyme inhibitor assessed by acoustic densitometry. J Cardiovasc Pharmacol 2000; 36: 361-8

19. Cardinale D, Colombo A, Sandri MT, Lamantia G, Colombo N, Civelli M et al. Prevention of high-dose chemotherapy-induced cardiotoxicity in high-risk patients by angiotensin-converting enzyme inhibition. Circulation 2006; 114: 2474-81 
20. Wouters KA, Kremer LCM, Miller TL, Herman EH, Lipshultz SE. Protecting against anthracyclines-induced myocardial damage: a review of the most promising strategies. Br J Haematol 2005; 131: 561-78

21. Youssef G, Links M. The prevention and management of cardiovascular complication of chemotherapy in patients with cancer. Am J Cardiovasc Drugs 2005; 5: 233-43

22. Felker GM, Thompson RE, Hare JM, Hruban RH, Clemetson DE, Howard DL et al. Underlying causes and long-term survival in patients with initially unexplained cardiomyopathy. N Engl J Med 2000; 342: 1077-84

23. Lefrak EA, Pitha J, Rosenheim S, Gotilieb JA. A clinicopathologic analysis of adriamycin cardiotoxicity. Cancer 1973; 32: 302-14

24. Cohen M, Kronzon I, Lebowitz A. Reversible doxorubicin-induced congestive heart failure. Arch Intern Med 1982; 142: 1570-1

25. Haq MM, Legha SS, Choksi J, Hortobagyi GN, Benjamin RS, Ewer M et al. Doxorubicin-induced congestive heart failure in adults. Cancer 1985, 56: 1361-5

26. Saini J, Rich MW, Lyss AP. Reversibility of severe left ventricular dysfunction due to doxorubicin cardiotoxicity. Report of three cases. Ann Intern Med 1987; 106: 814-6

27. Jensen BV, Nielsen SL, Skovsgaard T. Treatment with angiotensin-converting-enzyme inhibitor for epirubicin-induced dilated cardiomyopathy. Lancet 1996; 347: 297-9

28. Fazio S, Calmieri EA, Ferravate B, Bonè F, Biondi B, Saccà L. Doxorubicin-induced cardiomyopathy treated with carvedilol. Clin Cardiol 1998; 21: 777-9

29. Noori A, Lindenfeld J, Wolfel E, Ferguson D, Bristow MR, Lowes BD. Beta-blockade in adriamycin-induced cardiomyopathy. J Card Fail 2000; 6: 115-9

30. Jensen BV, Skovsgaard T, Nielsen SL. Functional monitoring of anthracycline cardiotoxicity: a prospective, blinded, long-term observational study of outcome in 120 patients. Ann Oncol 2002; 13: 699-709

31. Mukai Y, Yoshida T, Nakaike R, Mukai N, Iwato K, Kyo T et al. Five cases of anthracycline-induced cardiomyopathy effectively treated with carvedilol. Intern Med 2004; 43: 1087-8

32. Tallaj JA, Franco V, Rayburn BK, Pinderski L, Benza RL, Pamboukian S et al. Response of doxorubicin-induced cardiomyopathy to the current management strategy of heart failure.J Heart Lung Transplant 2005; 24: 2196-201

33. Tabet JY, Meurin P, Ben Driss A, Berthaux X, Weber H, Renaud N et al. Beta-blockade intolerance in anthracycline-induced cardiomyopathy. Int J Cardiol 2006; 106: 132-4

34. Cardinale D, Colombo A, Lamantia G, Colombo N, Civelli M, De Giacomi G et al. Anthracycline-induced cardiomyopathy. Clinical relevance and response to pharmacologic therapy. J Am Coll Cardiol 2010. In press.

\section{CORRESPONDING AUTHOR}

Dott.ssa Daniela Cardinale

Unità di Cardiologia

Istituto Europeo di Oncologia

Via Ripamonti 435

20141 Milano

Tel. 0257489539

Fax 02700509829

e-mail: daniela.cardinale@ieo.it 